\title{
Generatividad y Satisfacción Vital en un una muestra de abuelos/as de Santiago, Chile
}

\author{
Generativity and Life Satisfaction in a sample of grandparents from \\ Santiago, Chile
}

\author{
M. Elena Larrain \\ Beatriz Zegers \\ Yasna Orellana \\ Escuela de Psicología, Universidad de los Andes, Chile \\ (Recibido: 30 de junio de 2017) (Aceptado: 12 de agosto de 2019)
}

\begin{abstract}
Resumen
Se presentan los resultados de una muestra de 385 abuelos 1 chilenos estratificada por sexo, edad y nivel de ingreso del grupo familiar en la Escala de Generatividad de Loyola (McAdams \& de St. Aubin, 1992) adaptada para abuelos en Chile (EGL-A) por Larrain, Zegers \& Orellana (2016) y en el Î́ndice de Satisfacción Vital de Neugarten, Havighurst \& Tobin $(1961,1996)$ en su versión adaptada a Chile por Zegers, RojasBarahona \& Föster (2009). No se encontró evidencia de correlación positiva entre los puntajes totales de ambos instrumentos, pero sí al estratificar por escolaridad, la correlación entre generatividad y la dimensión congruencia del LSI-A resultó positiva para quienes poseían escolaridad media $(r=0.215, p=0.021) \mathrm{o}$ superior $(\mathrm{r}=0.230, \mathrm{p}=0.0066)$.

Palabras clave: abuelos, nietos, generatividad, satisfacción vital
\end{abstract}

\begin{abstract}
The results of a sample of 383 chilean grandparents stratified by sex, age and income level to the Loyola Generativity Scale (LGS) (McAdams \& de St. Aubin, 1992) adapted for grandparents in Chile (EGL-A) by Larrain, Zegers \& Orellana (2016) and the Life Satisfaction Index Neugarten, Havighurst \& Tobin (1961, 1996) in its adapted version to Chile by Zegers, Rojas-Barahona \& Föster (2009) are presented. It was not found a positive correlation between the total scores of both instruments but it was positive when the sample was stratified by school level between generativity and the congruence dimension of the LSI-A for all those with complete high school studies $(\mathrm{r}=0.215, \mathrm{p}=0.021)$ or college studies $(\mathrm{r}=0.230, \mathrm{p}=0.0066)$.

Key words: grandparents, grandchildren, generativity, life satisfaction
\end{abstract}

1 Nota: Las autoras asumen la importancia de la distinción lingüística de género; sin embargo, para facilitar la lectura del texto, en adelante esta será obviada, tanto para referirnos a los abuelos, a los hijos como a los nietos.

Correspondencia: M. Elena Larrain melarrain17@gmail.com. Dirección Postal: Avda. Álvaro del Portillo 12455, Las Condes, Santiago, Chile

Nota del Autor: Este trabajo ha sido elaborado en el marco del proyecto de investigación PSI-OC: IN0GTO201258, Abuelidad, generatividad y satisfacción vital financiado por el Fondo de Ayuda a la Investigación de la Universidad de los Andes. 


\section{Introducción}

Se afirma que el rol de abuelo es un rol sin rol (Clavan, 1978 en Pinazo, 1999), un rol tenue o ambiguo al no existir claras prescripciones en relación a los derechos y obligaciones que le corresponden (Silverman, 1982, Wood, 1982 en Silverstein, Giarusso \& Bengtson, 1998). Con todo, existen diversas facetas del rol de abuelo que incluyen los niveles actitudinal, conductual, afectivo y simbólico (Pinazo, 1999). El desempeño del mismo tiene variadas repercusiones al interior de la familia, incluyendo a los abuelos mismos. En Chile, se trata de una realidad poco estudiada. Solo hemos conocido algunos antecedentes. Un estudio en una muestra representativa de adultos mayores (AM) chilenos, indica que: a) la mayoría de las personas mayores mantiene contacto con sus nietos al menos una vez a la semana, b) el $65.7 \%$ se siente muy satisfecho de la relación con sus nietos y, c) las mujeres y quienes tienen mayor nivel educacional se sienten más satisfechos con la relación que tienen con sus nietos (Instituto de Sociología UC, 2010, pp. 88-89).

La figura del abuelo desempeña un importante papel para las relaciones familiares, en especial en un tiempo en que la familia está sometida a cambios significativos. La importancia de la generatividad en la Adultez Madura y Adultez Mayor ha sido señalada por Erikson (1976, 2000), siendo un concepto importante en el desarrollo humano. Su estudio no ha recibido la atención debida en gerontología, pero la investigación en esta área es promisoria (Schoklitsch \& Baumann, 2011).

La satisfacción vital corresponde a una valoración subjetiva del bienestar, siendo de particular interés en las personas que están envejeciendo cuando se quiere evaluar su salud subjetiva. Explorar la relación entre la generatividad desplegada como abuelo y la satisfacción vital puede contribuir a comprender mejor los aportes que estos últimos pueden hacer a favor del desarrollo de las generaciones futuras y a uno de los roles relevantes para que los AM vivan bien los años que tienen por delante.

El objetivo del estudio fue explorar la existencia de una relación entre satisfacción vital y generatividad en abuelos y variables sociodemográficas como edad, sexo y niveles de ingreso.

\section{Abuelidad, Generatividad y Satisfacción Vital}

En el mundo actual, las personas pueden esperar dedicar varias décadas de su vida al rol de abuelo. Durante estos años posiblemente ejerzan simultáneamente diversos roles y estén empleados o activos en muchas áreas de su vida. El rol del abuelo se desarrolla actualmente de modo diverso a épocas anteriores. Tanto en USA como en Europa cerca del 50\% de los abuelos proveen algún tipo de cuidado a sus nietos (Glaser et al., 2013). En Chile, la gran mayoría de los AM vive acompañado y un 38\% lo hace con nietos; el $23.8 \%$ refiere estar a cargo de ellos a diario o varias veces por semana, cifra menor en un 5.5\% respecto a la III Encuesta sobre Calidad de Vida en la Vejez-2013 (Instituto Sociología UC, 2014), las mujeres menores de 75 años y con educación media son quienes asumen mayoritariamente este cuidado (Programa Adulto Mayor UC - Centro UC Estudios de Vejez y Envejecimiento, 2017). La citada encuesta indica que en 6 años "ha disminuido más de 10 puntos porcentuales la percepción de obligatoriedad en el cuidado de los nietos" (Programa Adulto Mayor UC - Centro UC Estudios de Vejez y Envejecimiento, 2017, p. 86). El estudio de Powdthavee, (2011) que incluye 11 países europeos, reporta que en Europa las abuelas en particular, juegan un rol central en el cuidado de los nietos, sea este intenso u ocasional. En la citada investigación, el 44\% de los abuelos reportaron otorgar cuidados a sus nietos en ausencia de sus padres, sin que los padres de los niños estuviesen presentes. Glaser et al,. (2013) indican que las abuelas más jóvenes, sanas y con nietos más pequeños son quienes más probablemente provean de su cuidado. Blasco \& Salomone (2016) señalan que las mujeres españolas no habrían podido formar parte, como lo han hecho, del mercado laboral si no hubiera sido por las abuelas. La satisfacción en el desempeño del rol requiere de mayor estudio especialmente en estos casos.

Se conoce bastante sobre los beneficios que el apoyo cotidiano, el cuidado y seguridad fuera de la jornada escolar reportan para los nietos (por ej. Coall \& Hertwig, 2011); la influencia de aprendizajes informales (Rodriguez, 2015).

El estudio de los beneficios que el desempeño del rol de abuelo reporta a la persona misma ha explorado las asociaciones entre aspectos selectos de la abuelidad y el bienestar psicológico (Muller \& Litwin, 2011). De acuerdo a estos autores, la teoría del rol y la de la identidad son las perspectivas centrales en la actualidad. El marco conceptual desarrollado por Kivnick (1983), en el que la centralidad del rol de abuelo es la primera de 
cinco dimensiones usadas para explorar el auto-concepto y el significado del rol también es un paradigma teórico que ampara muchas de las investigaciones realizadas que se han realizado. Las restantes dimensiones descritas por esta autora, aluden al valor del anciano (traspasar la tradición y ser valorado en ese rol), la inmortalidad del clan, es decir la responsabilidad patriarcal o matriarcal, la identificación con los nietos y la inmortalidad familiar; el reinvolucramiento con el pasado personal que los hace revivir experiencias previas e identificarse con sus propios abuelos, los abuelos reviven sus vidas previas y se identifican con sus propios abuelos y por último; las actitudes de benevolencia hacia los nietos.

El estudio de la abuelidad desde la perspectiva de 'la generatividad' puede establecer como punto de partida que "la disponibilidad de abuelos a los nietos ofrecerá nuevos tipos de desafíos a la generatividad de los ancianos" (Peterson, 2002, p. 203). La generatividad en esencia es: "la preocupación por establecer y guiar a la nueva generación" (Erikson, 1976, p. 240). Existen diversas maneras de desarrollar esta tarea (procreando, cuidando de los más jóvenes, produciendo, creando, participando en la comunidad (de St. Aubin, McAdams \& Kim, 2004) pero implica la conciencia de la responsabilidad personal hacia la sociedad, los más jóvenes, débiles y con menos experiencias o subordinados en algún sentido (McAdams, de St. Aubin \& Logan, 1993). K. Erikson (2004) plantea que la generatividad es más compleja de lo que se piensa, y específica que desde una perspectiva sociológica, corresponde primariamente a los padres con el fin de proteger su legado a fin de que éste no se extinga. El concepto de generatividad cultural, hace referencia al interés de los adultos por trasmitir los instrumentos e ideas propios de su cultura a las siguientes generaciones, relacionado al bienestar psicológico (McAdams \& Logan, 2004). Kotre $(1984,2004)$ distingue dos tipos de generatividad: la comunitaria, que implica nutrir y cuidar de otros a través de los vínculos y, la agéntica que se refiere al fortalecimiento del yo mediante el liderazgo, la producción o la actividad creativa. Cheng, (2009) indica que la investigación sugiere que la generatividad puede ser el único y más importante factor en el logro de la integridad yoica, aspecto relevante en la madurez personal. La preocupación generativa en este estudio, se reporta como algo que se asocia de manera moderada a fuerte con el bienestar psicológico, en tanto que las acciones concretas no aparecen a pesar de la fuerte correlación con la preocupación generativa (Grossbaum \& Bates, 2002; Mc Adams, de St. Aubin \& Logan, 1993). Como señala Cheng (2009), dada la importancia de la generatividad en el desarrollo de la vida tardía, no es soprendente prediga el bienestar en estas edades. Sin embargo, precisa que, la relación entre los actos generativos y el bienestar puede ser algo muy complejo. Por ejemplo, proveer cuidado a los nietos y ser generativo puede conllevar efectos beneficiosos para los abuelos tales como tener un estilo de vida más activo, mejor salud y comportamientos asociados (Hughes, Waite, LaPierre \& Luo, 2007) y una mayor satisfacción vital generalizada (Powdthavee, 2011). Para Villar (2013), el reto es encontrar un concepto unificador que permita entender y estudiar el buen envejecimiento tanto desde la perspectiva individual como social, concepto que para él sería el de generatividad. Éste establece metas de desarrollo basadas en los aportes que los AM realizan en los contextos sociales en que participan. Para este autor, la generatividad, a su vez, enmarca esas contribuciones dentro de un esquema de desarrollo individual que favorece las ganancias y beneficios personales posibles en la última etapa de la vida. Con todo para Arpino \& Bordone (2014), aun cuando el abuelo sea generativo, también puede vivir su rol como algo estresante, que limita sus oportunidades de involucramiento en otros roles sociales y de autocuidado con los consiguientes efectos negativos.

Estudios sobre la abuelidad relacionan las actividades desarrolladas con los nietos como una dimensión del comportamiento muy importante en el desempeño del rol de abuelo y en la satisfacción con el mismo (Hayslip \& Kaminski, 2005; Reitzes \& Mutran 2004; Thiele \& Whelan 2008). Reitzes \& Mutran (2004) encontraron diferencias entre abuelos y abuelas respecto a la centralidad del rol. La importancia otorgada al rol se relacionaba positivamente con la frecuencia del contacto para los abuelos, no así para las abuelas. No obstante, la satisfacción con el rol de abuelo estaba relacionada de manera igualmente positiva para ambos sexos. Concluyeron que se requiere de mayor investigación respecto a las expectativas y la experiencia del rol de abuelo. Baydar \& Brooks-Gunn, (1998) relacionan la diversidad de roles además del rol de abuelo con el bienestar psíquico. La multiplicidad de roles otorgaría mayor satisfacción y bienestar psicológico debido a un aumento en los sentimientos personales de poder, de tener recursos y también de gratificación emocional. Este estudio reveló que las abuelas que desempeñaban muchos roles simultáneamente y que estaban altamente involucradas con ellos, no presentaron señales de que su bienestar psicológico estuviese comprometido. Pero desde la perspectiva contraria, es decir, que con la edad los roles generan estrés e imponen presión al individuo, el rol de abuelo no necesariamente se vincularía con bienestar debido al conflicto de roles y a las demandas involucradas que 
reducen el nivel de satisfacción psicológica (Muller \& Litwin, 2011). Coall \& Hertwig (2011) ; Pollet, Nettle, \& Nelissen (2007) también encontraron que las abuelas maternas son quienes más invierten en el rol. Coall \& Herwtig (2011) observaron que ellas son las que tienen más contacto con sus nietos, seguidas de los abuelos maternos, las abuelas paternas y finalmente los abuelos paternos. También encontraron que el involucramiento de los abuelos maternos en el cuidado de los nietos tiende a ser mediado por el compromiso de sus esposas en él. La satisfacción con el rol de abuelo es por tanto un aspecto complejo y multifacético vinculado a diversas variables como sexo, edad, salud física, ocupación, etc.

En síntesis, los abuelos tienden a involucrarse frecuentemente con los nietos y estas interacciones se caracterizan por satisfacción y cercanía. Existen estudios que concluyen que los abuelos que pierden contacto con sus nietos experimentan un impacto negativo en su salud emocional (Drew \& Silverstein 2007). Sin embargo, la literatura no es concluyente. El estudio de Muller \& Litwin (2011) no encontró la asociación hipotetizada, por el contrario observaron que los abuelos que tenían un contacto más frecuente, estaban menos involucrados en otros roles sociales, eran quienes desplegaron mayores síntomas depresivos, lo mismo que quienes expresaron alto cumplimiento con las afirmaciones sobre los deberes y quienes consideraban el rol como algo de importancia. La centralidad del rol de abuelo en la vida de la persona que respondía no predecía su bienestar psicológico. El predictor más fuerte fue el status físico de la persona: mientras mayores eran las limitaciones, más síntomas depresivos, menos bienestar o satisfacción. Herrera, Barros \& Fernández (2011), en Chile, establecieron que el factor con mayor poder predictivo en la calidad de vida de los AM, era la capacidad funcional que permitía la actividad física y para la satisfacción, entre los factores más importantes se encontraba el sentimiento de autoeficacia y la buena calidad de las relaciones sociales. El sexo y el nivel de ingreso fueron los otros dos predictores más importantes siendo las mujeres y quienes tenían menos ingresos las que experimentaban niveles más bajos de bienestar. Muller \& Litwin (2011), encontraron que la edad y el nivel educacional no se relacionaron de manera significativa con el bienestar. Una posible explicación a estos hallazgos la ofrecen Gauthier (2002) y Silverstein \& Marenco (2001) quienes señalan que los abuelos modernos en USA tienen muchas de las características de la clase media, son más sanos, prósperos y educados que los abuelos de la generación previa. Valoran la independencia personal y el involucramiento social fuera de la red familiar y tienden a apoyarse en fuentes extra familiares tales como el lugar de trabajo y los círculos sociales para su sentido de valor propio. Es decir, en la actualidad existen valores que compiten y reemplazan aquellos basados en la solidaridad familiar (Silverstein, Giarusso \& Bengston, 1998).

La generatividad desplegada por los abuelos y su relación con la satisfacción subjetiva depende de cómo se explore y arroja asociaciones no unívocas. Cheng (2009), señala que el efecto de las acciones generativas en los años tardíos de la vida sobre el bienestar, se encuentra mediado por el respeto que los AM perciben de las generaciones jóvenes. La relación entre generatividad y satisfacción vital pudiese también estar mediada por la percepción de los abuelos del respeto que los nietos les manifiestan. Estos autores encontraron que cuando las actitudes son desfavorables surge un espiral descendente del desarrollo de la generatividad y del bienestar posible.

La relación entre abuelidad, generatividad y satisfacción vital no ha sido establecida de modo claro y se emplean diversos instrumentos para evaluarla. Moyano, Flores \& Soromaa (2011) plantean que los conceptos de felicidad y bienestar subjetivo son los componentes psicológicos de un concepto sociológico mayor: calidad de vida, asociado a bienestar subjetivo, bienestar psicológico, satisfacción vital y felicidad.

La satisfacción vital es un constructo psicológico que refleja el bienestar del individuo y fue introducido por Neugarten, Havighurst \& Tobin (1961). Puede investigarse centrándose en las conductas manifiestas o en la apreciación subjetiva que el sujeto realiza respecto de su vida actual y pasada, su felicidad y satisfacción. Esta última visión es la que Neugarten et al., $(1961,1996)$ emplearon como constructo a la base del Indice de Satisfacción Vital-A (LSI-A). La complejidad del constructo se refleja en la "paradoja del bienestar" que alude a las bajas correlaciones entre condiciones objetivas de calidad de vida y el bienestar subjetivo en adultos de todas las edades (Covinsky et al., 1999 en Inga \&Vara, 2006; Pennex et al., 1998 en Inga \& Vara, 2006; Staudinger, Fleeson \& Baltes, 1999). Smith, Borchelt, Maier \& Jopp (2002) recomiendan incorporar medidas de evaluación subjetiva de bienestar como es el LSI-A (Neugarten, Havighurst \& Tobin, 1961, 1996) en estudios sobre el envejecimiento. En Chile, usando otras medidas de evaluación, Herrera, Barros \& Carrasco (2012), encontraron los mayores niveles de satisfacción vital en AM de la 3ra. Edad (81\% en el grupo de 65-69 años y $75 \%$ en el de $70-74$ años). 


\section{Método}

\section{Participantes}

La población corresponde a abuelos autovalentes o con dependencia moderada evaluados mediante la escala de Actividades Instrumentales de la Vida Diaria (AIVD) (Lawton-Brody, 1969 en Marín, Gac \& Carrasco, 2006) de la Región Metropolitana (RM) de Santiago de Chile. Los criterios de inclusión fueron: sexo (Triadó, Martinez \& Villar, 2000; Delerue \& Borges, 2012); nivel de ingreso familiar: alto, medio y bajo (Encuesta de Caracterización Socioeconómica Nacional CASEN, 2009, Chile Ministerio de Desarrollo Social, 2011) (Hirshorn, 1998) y edad (45-59 y 60-75), (Baltes \& Smith, 2003; Cherlin \& Fustenberg, 1992; McAdams \& Logan, 2004; Neugarten, 1974/1996; Thomas, 1989 en Triadó, Martinez \& Villar, 2000; Stewart \& Wandewater, 1998).

El estudio incluyó 385 participantes: edad promedio 60.18 años $(\mathrm{DE}=7.99)$, 59.69 años en las mujeres $(\mathrm{DE}=$ 8.37) y 60.70 años en los hombres $(\mathrm{DE}=7.54)$ sin diferencias significativas $(\mathrm{T}(383)=1.25, \mathrm{p}=0.214)$. Nivel de ingreso: $37.53 \%$ ingreso bajo, $31.49 \%$ medio y $30.98 \%$ alto. Estado civil: $65.97 \%$ casados, $15.32 \%$ separados/ divorciados, $8.83 \%$ viudos y $9.87 \%$ solteros. Escolaridad: $0.52 \%$ sin escolaridad, $28.31 \%$ enseñanza básica completa, $35.32 \%$ enseñanza media completa y $35.86 \%$ estudios técnicos o universitarios. Trabajaban: $77.92 \%$ y $21.09 \%$ realizaban trabajos voluntarios. (Tabla 1 )

$16.88 \%$ tuvo su primer nieto antes de los 40 años, $4.94 \%$ cumplidos los 60 años y $78.18 \%$ entre los 40-59. El $31.33 \%$ vivía con algún nieto (abuelas $37.06 \%$ y abuelos $25.27 \%$ ) con diferencias significativas $(\mathrm{Z}=-2.49$, $\mathrm{p}=0.013$ ). (Tabla 2)

Hubo un porcentaje significativamente más alto de abuelos de ingreso medio y bajo que vivían con algún nieto y un porcentaje mayor de quienes tuvieron su primer nieto antes de los 40 años fueron abuela/os de ingreso alto. Los promedios de edad de los nietos menores fueron significativamente distintos entre los tres niveles. La edad del nieto mayor fue significativamente menor en el nivel de ingreso bajo comparado con los otros dos niveles. (Tabla 3)

\section{Instrumentos}

» Escala de Generatividad de Loyola (McAdams \& de St. Aubin, 1992) adaptada para abuelos EGL-A por Larrain, Zegers \& Orellana (2017) ${ }^{2}$. Consta de 13 ítems con 3 alternativas de respuestas tipo Lickert que evalúan generatividad general y no específica. El puntaje teórico esperado fluctúa entre 0 y 39 . A mayor puntaje mayor generatividad. El Análisis Factorial Confirmatorio (AFC) identificó dos factores: Generatividad Positiva y Dudosa como en el instrumento original. El primer factor explica un $72.39 \%$ y el segundo un $27.61 \%$ de la varianza total. La consistencia interna para Generatividad Positiva fue $\alpha=0.85$ y para Generatividad Dudosa según el coeficiente de Spearman-Brown fue de 0.79 (Eisinga, Grotenhuis \& Pelzer, 2013), ambas consideradas buenas (Henerson, Lyons \& Taylor, 1987). Los coeficientes Alpha de Cronbach para los ítems que conforman el factor 1 mostraron diferencias significativas $(\mathrm{Chi}(1)=5.67, \mathrm{p}=0.02)$ entre hombres $(0.87)$ y mujeres $(0.81)$. No se encontraron diferencias significativas según edad $(\mathrm{Chi}(1)=0.43$, $\mathrm{p}=0.51)$ ni según nivel de ingreso $(\mathrm{Chi}=2.71 \mathrm{DF}=2 \mathrm{p}=0.26)$.

» Índice de Satisfacción Vital (LSI-A) de Neugarten, Havighurst \& Tobin $(1961,1996)$ adaptada para Chile por Zegers, Rojas-Barahona y Förster (2009): evalúa la apreciación subjetiva del bienestar psicológico. La versión utilizada consta de 19 ítems y tiene 3 alternativas de respuestas. El puntaje total fluctúa entre 0 y 19 (a mayor puntaje mayor satisfacción vital). Su aplicación toma entre 10-15 minutos. La validez de constructo de la escala para Chile estimada a través el Análisis Factorial de Componentes Principales es de $50.62 \%$ e identificó cinco factores: congruencia entre logros deseados y obtenidos, entusiasmo, tono emocional, auto-

2 Para mayores detalles del estudio del instrumento, se remite al lector al artículo "Traducción y Adaptación de una Escala para Evaluar Generatividad en Abuelos en Santiago de Chile”, Larrain, Zegers y Orellana (2017). 
concepto, resolución y fortaleza. La validez concurrente con la Escala de Autoestima de Rosenberg (1965), validada para Chile por Rojas-Barahona, Zegers y Förster (2009) es de $r=0.455$ y significativa $(\mathrm{p}<0.01)$. Su consistencia interna es de $\alpha=0.80$.

» Escala de Actividades Instrumentales de la Vida Diaria (AIVD) de Lawton-Brody (1969, en Marín, Gac \& Carrasco, 2006): evalúa autonomía física en AM. Se aplicó la versión del Programa de Geriatría y Gerontología, Pontificia Universidad Católica de Chile (Marín, Gac y Carrasco, 2006) que toma entre 10 y 15 minutos. La confiabilidad interjueces fue de 0.85 . Su validez concurrente se ha estimado con escalas de valoración cognitiva (por ej., Mini Mental State Examination-MMSE). Su puntuación es dicotómica (1=autonomía, $0=$ dependencia). El puntaje final se clasifica en tres categorías: independencia total ( 8 puntos), dependencia moderada (4-7 puntos) y dependencia intensa (0-3 puntos).

» Cuestionario datos socio-demográficos: incluye fecha de nacimiento, edad, sexo, actividad laboral o de voluntariado, nivel de ingreso del grupo familiar, estado civil, escolaridad, número y sexo de los hijos y nietos, edad de los nietos, nieto hijo de padres separados, o nieto que viviese con él/ella, y edad a la que fue abuelo por primera vez.

\section{Procedimiento}

Todos los participantes firmaron el Consentimiento Informado aprobado por el Comité de Ética de la Facultad de Medicina de la Universidad de los Andes. Los instrumentos fueron administrados por alumnos o titulados de la Escuela de Psicología de la Universidad de los Andes, previamente capacitados por el equipo investigador.

\section{Análisis de Datos}

Se realizaron tablas de frecuencia, medidas como cuartiles, mínimos y máximos en el caso de variables cualitativas, nominales u ordinales. Se obtuvieron en algunos casos el promedio y desviación estándar. Para la asociación de variables cualitativas se utilizó test de Chi-cuadrado. Se aplicaron las pruebas no paramétricas de Mann Whitney o Kruskal Wallis cuando correspondiera. Las comparaciones posthoc se realizaron con el test de Bonferroni, las correlaciones mediante coeficiente de Spearman. Todos los análisis se hicieron usando el software STATA v.14 y el nivel de significación fue de $\alpha=0.05$

\section{Resultados}

\section{Escala de Generatividad para abuelos (EGL-A)}

La puntuación total para esta muestra fluctuó entre 3 y 39 puntos, con un puntaje mediano de 25 , percentil 25 de 20 y percentil 75 de 30 . Hubo diferencias significativas según sexo $(Z=-4.64, p=0.000)$ siendo más altos los puntajes en las mujeres $(\mathrm{Md}=27, \mathrm{Q} 1=23, \mathrm{Q} 3=31)$ que en los hombres $(\mathrm{Md}=23, \mathrm{Q} 1=19, \mathrm{Q} 3=29)$. Según nivel de ingreso familiar, los menos generativos fueron los con menor ingreso $(\mathrm{Md}=24, \mathrm{Q} 1=19, \mathrm{Q} 3=29)(\mathrm{Rank}=35.33$, $\mathrm{p}=0.004)$ comparados con los de ingreso medio $(\mathrm{Md}=26, \mathrm{Q} 1=21, \mathrm{Q} 3=31)($ Rank=42.81, $\mathrm{p}=0.001)$, sin diferencias entre los puntajes de nivel de ingreso medio y alto $(\mathrm{Md}=26, \mathrm{Q} 1=22, \mathrm{Q} 3=31)$, $(\operatorname{Rank}=7.49, \mathrm{p}=0.301)$. Por grupo etario no se encontraron diferencias significativas $(Z=-0.21, p=0.834)$.

Se encontraron diferencias significativas entre aquellos con educación básica y superior (Rank $=38.74$, $\mathrm{p}=$ 0.003 ), y educación media y superior ( $\operatorname{Rank}=37.65, \mathrm{p}=0.003$ ), siendo menos generativos los abuelos con educación técnico/superior. Respecto a los nietos, hubo diferencias entre los abuelos que viven con nietos $(\mathrm{Z}=-3.21, \mathrm{p}=0.001)(\mathrm{Md}=27, \mathrm{Q} 1=22, \mathrm{Q} 3=31)$ y los que no $(\mathrm{Md}=25, \mathrm{Q} 1=19, \mathrm{Q} 3=29)$, siendo más generativos los primeros. Los abuelos que señalaron tener nietos de hijos separados tuvieron un puntaje significativamente mayor $(\mathrm{Md}=28, \mathrm{Q} 1=24, \mathrm{Q}=31)(\mathrm{Z}=-5.22, \mathrm{p}=0.000)$ que aquellos que no $(\mathrm{Md}=24, \mathrm{Q} 1=19, \mathrm{Q} 3=29)$. Los que obtuvieron puntajes más altos en generatividad fueron los abuelos que tenían nietos de hijos separados y que vivían con alguno $(\mathrm{Md}=29, \mathrm{Q} 1=25, \mathrm{Q} 3=32)(\mathrm{Z}=-1.991, \mathrm{p}=0.047)$. 
Los abuelos que tenían más de tres nietos obtuvieron puntajes mayores $(\mathrm{Md}=27, \mathrm{Q} 1=22, \mathrm{Q} 3=30)$ (Rank=28.38, $\mathrm{p}=0.017)$ que los con dos o tres nietos $(\mathrm{Md}=25, \mathrm{Q} 1=20, \mathrm{Q} 3=29)$ y aún mayores que aquellos con solo un nieto $(\mathrm{Rank}=38.17, \mathrm{p}=0.004)(\mathrm{Md}=25, \mathrm{Q} 1=19, \mathrm{Q} 3=29)$, sin haber diferencia entre éstos dos últimos $($ Rank $=9.79, p=0.262)$.

No existieron diferencias entre los abuelos cuyo nieto mayor tenía menos de 10 años y aquellos cuyos nietos eran mayores de 10 años $(Z=0.67, p=0.501)$. (Tabla 4)

\section{Índice de Satisfacción Vital (LSI-A) adaptado a población chilena}

La puntuación total fluctuó entre 2 y 19 puntos, con un puntaje mediano de 14 puntos, percentil 25 de 12 y percentil 75 de 16. Hubo diferencias significativas según sexo $(Z=-2.56, p=0.011)$, las mujeres puntuaron más alto $(\mathrm{Md}=15, \mathrm{Q} 1=12, \mathrm{Q} 3=17)$ que los hombres $(\mathrm{Md}=13, \mathrm{Q} 1=11, \mathrm{Q} 3=16)$. El puntaje de aquellos con mayor ingreso $(\mathrm{Md}=16, \mathrm{Q} 1=14, \mathrm{Q} 3=18)$ fue superior $(\mathrm{Rank}=97.74, \mathrm{p}=0.000)$ a los de ingreso medio $(\mathrm{Md}=13, \mathrm{Q} 1=11$, $\mathrm{Q} 3=15)$ y $($ Rank=108.49, $\mathrm{p}=0.000)$ a los de ingreso bajo $(\mathrm{Md}=13, \mathrm{Q} 1=11, \mathrm{Q}=15)$, sin diferencia entre estos últimos (Rank=10.75, $\mathrm{p}=0.228$ ). No hubo diferencias significativas por grupo etario $(\mathrm{Z}=-0.15, \mathrm{p}=0.882)$. Los sujetos con educación superior puntuaron más alto que los de educación media (Rank=71.98, $\mathrm{p}=0.000$ ) y básica (Rank=111.58, p=0.000). Éstos últimos obtuvieron menores puntajes que los abuelos con educación media (Rank=39.60, $\mathrm{p}=0.003$ ).

\section{Análisis de correlaciones entre la EGL-A y LSI-A}

No se encontró correlación entre los puntajes totales de ambos instrumentos $(r=0.013, p=0.807)$. Se observó correlación positiva solo entre el factor congruencia entre objetivos deseados y alcanzados de la LSI-A y el factor generatividad positiva de la EGL-A $(\mathrm{r}=0.12, \mathrm{p}=0.020)$. Al estratificar por sexo, ésta aumenta en los hombres $(r=0.23, p=0.002)$ y desaparece en las mujeres. También resultó positiva para abuelos con escolaridad media $(r=0.27, p=0.001)$ o técnico/superior $(r=0.20, p=0.011)$, no así en aquellos con baja escolaridad ( $r$ $=-0.09, \mathrm{p}=0.346$ ).

Al estratificar por sexo, ajustar por escolaridad y grupo etario, se observó una correlación positiva y significativa $(\mathrm{r}=0.44, \mathrm{p}=0.005)$ entre el factor generatividad positiva y el factor congruencia sólo en hombres de escolaridad media y mayores de 60 años. En hombres de escolaridad técnico/universitario menores de 60 años $(\mathrm{r}=0.33, \mathrm{p}=0.055)$, dicha correlación no fue significativa debido al tamaño del subestrato $(\mathrm{n}=35)$. En la dimensión resolución y fortaleza en el caso de las mujeres de escolaridad media y menores de 60 años la correlación fue de $\mathrm{r}=0.35(\mathrm{p}=0.050)$ pero sin significación estadística $(\mathrm{n}=33)$. Lo anterior también es válido para la dimensión autoconcepto $(\mathrm{r}=-0.37, \mathrm{p}=0.058)$ en el subestrato de hombres de escolaridad básica y menores de 60 años (n=27).

Se observó que los abuelos con escolaridad media y técnico/universitario que trabajaban obtuvieron correlaciones entre el factor congruencia y generatividad positiva de $\mathrm{r}=0.37(\mathrm{p}=0.001)$ y $\mathrm{r}=0.36(\mathrm{p}=0.009)$ respectivamente. En el caso de las abuelas con escolaridad técnica/universitaria y que trabajaban, se encontró una correlación negativa $(\mathrm{r}=-0.31, \mathrm{p}=0.030)$ entre el factor autoconcepto y generatividad positiva. (Tabla 5)

\section{Discusión y Conclusiones}

La novedad de este trabajo radica en que explora una dimensión específica y poco estudiada de la generatividad en Chile, a saber, la generatividad como abuelos y cuya importancia en la literatura científica ha sido progresiva. Los resultados con el instrumento EGL-A (Larrain, Zegers y Orellana, 2017), no son extrapolables al estudio de otras formas de generatividad. Por su parte, el constructo de satisfacción vital, es complejo y existen diversas perspectivas para su estudio (modelos objetivos, subjetivos, etc, Brown, Bowling y Flynn, 2004).

Dado que la muestra fue intencional, futuras investigaciones debiesen contar con muestras representativas y aleatorias. Si bien se cumplió el criterio del número mínimo de sujetos por celdilla, el promedio de edad fue de 60.18 años con variaciones no significativas según sexo y con baja representación en los extremos (solo un $10 \%$ de hombres y mujeres tenían menos de 48 y más de 72 años). Lo anterior podría ser un factor que explique 
que el $77.92 \%$ estaba laboralmente activo, aunque las abuelas mayores de 60 fueron menos que los hombres (50.5\% vs. $86.73 \%)$, lo cual posiblemente se modifique en futuras generaciones. La distribución según ingreso resultó homogénea por estrato, y por escolaridad hubo algo menos de sujetos en el estrato de baja escolaridad ( $28.31 \%$ vs. $35.32 \%$ ). El $16.88 \%$ de los abuelos de la muestra lo fue antes de los 40 años y $4.94 \%$ después de los 59 años. La muestra estuvo conformada predominantemente por abuelos de nietos pequeños y edad escolar (promedio de edad del nieto mayor 11.61 años y del menor, 4.08 años) con mayores desviaciones estándar entre los mayores. Esta distribución se asociaría a un modo más cercano de ser abuelo (i.e. Cherlin \& Fustenberg, 1992; Thomas, 1989 en Triadó, Martínez \& Villar, 2000) existiendo menores antecedentes empíricos de la relación con nietos adolescentes. Un mayor número de mujeres señaló vivir con algún nieto ( $37.06 \%$ vs. 25.27\% de los hombres). Según ingreso, el porcentaje mayor de abuelos que indicaron vivir con algún nieto se encuentra en el nivel medio (48.18\%).

Si bien se esperaba siguiendo a autores como Powdthavee (2011) y Cheng (2009), no se encontró una correlación positiva entre la generatividad de abuelos y la satisfacción vital. No obstante, Cheng (2009) precisa que la relación entre ambas puede ser muy compleja y se asociaría entre otros a la edad, visión compartida por Villar (2013), quien relaciona la generatividad con las posibles ganancias en la última etapa de la vida. Por otro lado, la variable generatividad no es discreta, ni emerge en un momento o a una edad determinada en la vida del sujeto sino más bien es una que se va enriqueciendo, diferenciando y profundizando en el curso de la vida y que Stewart \& Wandewater, (1998) han denominado como trayectoria generativa. Asimismo, Arpino \& Bordone (2014) describen que la generatividad como abuelo también puede conllevar un cierto monto de estrés al limitar la participación en otros roles sociales y de autocuidado, asunto que no se estudió en la presente investigación.

De las correlaciones presentadas en el análisis de resultados, se destaca aquí aquella positiva y significativa entre generatividad positiva y el factor congruencia de LSI-A que surge al estratificar por sexo, ajustar por escolaridad y grupo etario solo en el subestrato de hombres de escolaridad media y mayores de 60 años. Posiblemente el hecho de que no aparezca esta correlación en mujeres pueda comprenderse en parte si se considera que a partir de mediados del siglo XX las condiciones de vida para las mujeres han variado significativamente, introduciendo diferencias entre cohortes. Por otra parte, el que no se haya encontrado correlación en el subestrato de hombres con baja escolaridad, es concordante con la literatura en torno a la satisfacción vital y el paso de los años (Lehr \& Thomae, 2009). Se sugiere para futuros estudios que la población de abuelos se estratifique según escolaridad más que según nivel de ingresos ya que se ha establecido que a largo plazo, la educación "determina" el nivel de ingreso (Contreras, 1998, p. 330); además esto es concordante con algunos de los hallazgos mencionados en la Segunda Encuesta Calidad de Vida en la Vejez (Instituto Sociología UC, 2010).

Debido a que los tamaños muestrales de algunos de los subestratos fueron "pequeños", algunas de las correlaciones encontradas, aunque fueron superiores (inferiores en el caso negativo) a 0.20 , no fueron significativas. Esto queda como una limitación a ser subsanada en estudios posteriores con muestras de mayor tamaño, para verificar si se mantienen dichos resultados.

$\mathrm{Al}$ analizar los resultados por separado de ambos instrumentos en relación a las variables de estratificación, aparecen hallazgos interesantes, algunos de los cuales se vinculan a las hipótesis específicas.

Otro hallazgo interesante es que quienes vivían con algún nieto y que además tenían nietos de hijos separados, puntuaron más alto en generatividad que aquellos que no vivían con nietos ni tenían nietos de hijos separados. Este resultado no es fácil de interpretar debido a que es posible que la generatividad sea una variable presente en este grupo de abuelos con antelación al hecho de vivir con los nietos lo cual, requeriría hacer un estudio retrospectivo en esos abuelos. Lo contrario puede a su vez también ser posible, a saber, que el vivir con nietos, constituye un estímulo para la generatividad. Pero en futuras investigaciones habría que explorar el estilo de ser abuelo (Neugarten \& Weinstein, 1964), toda vez que la LGS-A apunta a evaluar intereses generativos y no conductas generativas las que están más relacionadas con estos estilos. A su vez sería interesante relacionar la calidad de los vínculos y la genealogía del nieto-abuelo.

En relación al número de nietos, se encontró que tener más de tres nietos aumenta la generatividad sin que exista relación entre la generatividad y la edad de los nietos.

Respecto a satisfacción vital, el modo y los instrumentos que se utilicen para explorarla puede arrojar resultados contradictorios como lo relatan Glenn \& McLanahan (1982) quienes estudiaron la satisfacción marital reportada respecto a tener hijos. Si se les preguntaba a los sujetos, si los niños habían afectado sus vidas matrimoniales, la mayoría concluía que sí y de manera muy positiva. Sin embargo, frente a la pregunta de cuán 
satisfechos estaban con su matrimonio, ellos reportaban más bajos niveles de satisfacción marital que quienes no tenían hijos o ya no los tenían a su cuidado. Algo análogo puede ocurrir con la valoración subjetiva de la satisfacción vital del abuelo y su relación con generatividad, cuyas correlaciones no fueron de acuerdo a lo esperado en todas las dimensiones de la escala de SV.

Se encontró mayor satisfacción vital en mujeres que en hombres. En relación al nivel de ingreso, a mayor ingreso más satisfacción vital sin diferencias entre los niveles medio y bajo. No se encontró diferencias en satisfacción vital de acuerdo a la edad de los abuelos. En escolaridad se observó que la mayor satisfacción vital se encontraba en quienes poseían educación superior, seguidos por los de educación media. Los abuelos del nivel educacional más bajo fueron los menos satisfechos.

Al comparar los resultados de satisfacción vital en la presente investigación, con la validación del instrumento realizada en Chile con personas de 30-75 años (Zegers, Rojas-Barahona y Förster (2009) se observó que respecto a escolaridad los resultados son concordantes, es decir hay mayor satisfacción vital en los sujetos con mayor escolaridad. Análogamente tampoco hubo diferencias en relación a la variable edad. Considerando la variable sexo hubo disparidad entre ambos estudios. Lo anterior puede tener que ver con los procedimientos de muestreo.

Las limitaciones del estudio dicen relación con que la estratificación de la muestra solo consideró el nivel de ingreso del grupo familiar de todos los parámetros que emplea CASEN 2009 (Chile, Ministerio de Desarrollo Social, 2011). Esto no permitió una discriminación clara, al menos en el grupo de sujetos en el rango alto porque el punto de corte de este nivel es muy bajo (límite inferior $=\$ 424.169$ pesos).

Futuras investigaciones deberían preguntar específicamente por número de nietos de cada hijo, frecuencia del contacto abuelos-nietos, vivir solo o acompañado, variables todas que pueden ayudar a explicar algunos de los resultados. Respecto de los abuelos también sería interesante estudiar en profundidad el status físico y no solo la autonomía en relación a actividades de la vida diaria, salud mental general y percepción del grado de respeto que los nietos manifiestan a sus abuelos dado que son variables que se han asociado a calidad de vida, bienestar, satisfacción vital y generatividad como se estableció en el marco teórico.

Debido a que los tamaños muestrales de algunos de los subestratos eran "pequeños", algunas de las correlaciones encontradas, aunque fueron superiores (inferiores en el caso negativo) a 0.30 , no fueron significativas. Esto queda como una limitación a ser subsanada en estudios posteriores con muestras de mayor tamaño, para verificar si se mantienen dichos resultados.

En resumen, el estudio de la generatividad en los abuelos aparece como un marco de referencia promisorio a continuar profundizándose en el curso de los años adultos y de la AM. Especialmente en este último período, se requiere de un acopio de investigación, la que entregará información relevante respecto al grado en que el concepto de generatividad resulta enriquecedor en la comprensión de un envejecimiento exitoso (Villar, 2013).

\section{Referencias}

Arpino, B., \& Bordone, V. (2014). Does grandparenting pay off? the effect of child care on grandparents' cognitive functioning. Journal of Marriage and Family, 76, 337-351.

Baltes, P. B., \& Smith, J. (2003). New frontiers in the future of aging: From successful aging of the young old to the dilemmas of the fourth age. Gerontology, 49, 123-135. http://doi.org/10.1159/000067946

Baydar, N., \& Brooks-Gunn, J. (1998). Profiles of Grandmothers Who Help Care for Their Grandchildren in the United States. Family Relations, 47, 385-393. http://doi.org/ 10.2307/585269. Recuperado de: http://www.jstor.org/stable/585269

Blasco, M., \& Salomone, M. (2016). Morir joven, a los 140. El papel de los telómeros en el envejecimiento y las historia de cómo trabajan los cientificos para conseguir que vivamos más y mejor. Barcelona: Paidós.

Brown, J., Bowling, A., \& Flynn, E. (Eds.) (2004). Models of quality of life: a taxonomy overview and systematic review of the literature. FORUM Project, European Forum and Population Ageing Research, University of Sheffield, Sheffield.

Coall, D. A., \& Hertwig, R. (2011). Grandparental Investment A Relic of the Past or a Resource for the Future? Current Directions in Psychological Science, 20, 93-98. http://doi.org/10.1177/0963721411403269

Contreras, D. (1998) Distribución del ingreso en Chile. Nuevos hechos y algunos mitos. Perspectivas en Políticas, Economía y Gestión/ Universidad de Chile, Departamento de Ingeniería Industrial, 2, 311-332.

Cheng, S. T. (2009). Generativity in later life: Perceived respect from younger generations as a determinant of goal disengagement and 
psychological well-being. The Journals of Gerontology Series B: Psychological Sciences and Social Sciences, 64, 45-54. http://doi. org/10.193/geronb/gbn027

Cherlin, A. J., \& Fustenberg, F. F. Jr. (1992). The new American grandparents. A place in a family. A life apart. U. S. A.; Basic Book Inc. Chile, Ministerio de Desarrollo Social (2011). Indicadores y fuentes de información: Encuesta Casen (2009). Recuperado desde http:// observatorio.ministeriodesarrollosocial.gob.cl/indicadores/indicadores_fuentes.php

Delerue, A. \& Borges, R. (2012). Understanding adolescent grandchildren's influence on their grandparents. In S. Arber, \& V. Timonen (Eds.). Contemporary grandparenting. Changing family relationships in global contexts (pp. 203- 224). Great Britain: The Policy Press, University of Bristol.

de St. Aubin, E., McAdams, D. P., \& Kim, T. (2004). The generative society: An epilogue. In E. de St. Aubin, D. P. McAdams, \& T. Kim (Eds.). The generative society. Caring for future generations (pp. 265-271). Washington D.C.: American Psychological Association.

Drew, L. M., \& Silverstein, M. (2007). Grandparents' psychological well-being after loss of contact with their grandchildren. Journal of Family Psychology, 21, 372-379. http://doi.org/10.1037/0893-3200.21.3.372

Eisinga, R., Grotenhuis, M. T., \& Pelzer, B. (2013). The reliability of a two-item scale: Pearson, Cronbach, or Spearman-Brown? International Journal of Public Health, 5, 637-642. http://doi.org/10.1007/s00038-012-0416-3

Erikson, E. H. (1976). Infancia y sociedad. Buenos Aires: Editorial Hormé, S. A. E.

Erikson, E. H. (2000). El ciclo vital completado: Versión extendida. Buenos Aires: Editorial Paidós, S. A. I. C. F.

Erikson, K. (2004). Reflections on generativity and society: A sociologist's perspective. In E. de St. Aubin, D. P. McAdams, \& T. Kim,

T. (Eds.), The generative society. Caring for future generations (pp. 51-61). Washington: D.C. American Psychological Association.

Gauthier, A. (2002). The role of grandparents. Current Sociolog, 50, 295-307 http://doi.org/10.1177/0011392102050002623

Glaser, K., Price, D., Di Gessa, G., Ribe, E., Stuchbury, R., \& Tinker, A. (2013). Grandparenting in Europe: family policy and grandparents' role in providing childcare. Grandparents Plus, July, 2013. London.

Glenn, N. D., \& McLanahan, S. (1982). Children and marital happiness: A further specification of the relationship. Journal of Marriage and the Family, 44, 63-72. http://dx.doi.org/10.2307/351263

Grossbaum, M. F., \& Bates, G. W. (2002). Correlates of psychological well-being at midlife. The role of generativity, agency and communion, and narrative themes. International Journal of Behavioral Development, 26, 120-127. http://dx.doi.org/10.1080/01650250042000654

Hayslip, B., \& Kaminski, P. (2005). Grandparents raising their grandchildren: A review of the literature and suggestions for practice. The Gerontologist, 45, 262-269. https://doi.org/10.1093/geront/45.2.262

Herrera, S., Barros, C., \& Fernández, M. B. (2011). Predictors of quality of life in old age. A multivariate study in Chile. Journal of Population Ageing, 4, 121-139. http://doi.org/ 10.1007/s12062-011-9043-7

Herrera, S., Barros, C., \& Carrasco, M. (2012). Cohesión familiar, solidaridad intergeneracional y conflicto: Impacto en el bienestar del adulto mayor. (Proyecto Fondecyt 1060326). Comunicación personal.

Henerson, M. E., Lyons, L., \& Taylor, C. (1987). How to measure attitudes. University of California. Los Ángeles: Sage Publications.

Hirshorn, B. (1998). Grandparents as caregivers. In M. Szinovacz (Ed.), Handbook on grandparenthood (pp. 200-214). Westport, Connecticut, London: Greenwood Press.

Hughes, M. E., Waite, L. J., LaPierre, T. A., \& Luo, Y. (2007). All in the family: The impact of caring for grandchildren on grandparents' health. The Journals of Gerontology Series B: Psychological Sciences and Social Sciences, 62, https://doi.org/10.1093/geronb/62.2.S108

Inga, J., \& Vara, A. (2006). Factores asociados a la satisfacción de vida de adultos mayores de 60 años en Lima-Perú. Universitas Psychologica, Bogotá, Colombia, 5, 475-495.

Instituto de Sociología UC (2010). Chile y sus mayores: resultados Segunda Encuesta Nacional Calidad de Vida en la Vejez 2010. Santiago, Chile: Pontificia Universidad Católica de Chile/Servicio Nacional del Adulto Mayor/Caja Los Andes.

Instituto de Sociología UC (2014). Chile y sus mayores: resultados Tercera Encuesta Nacional Calidad de Vida en la Vejez 2013. Santiago, Chile: Pontificia Universidad Católica de Chile/Servicio Nacional del Adulto Mayor/Caja Los Andes.

Kivnick, H. Q. (1983). Dimensions of grandparenthood meaning: Deductive conceptualization and empirical derivation. Journal of Personality and Social Psychology, 44, 1056-1068. http://doi.org/10.1037/0022-3514.44.5.1056

Kotre, J. (1984). Outliving the Self: Generativity and the Interpretation of Lives. Baltimore: John Hopkins University Press.

Kotre, J. (2004). Generative and culture: What meaning can do. In E. de St. Aubin, D. P. McAdams, \& T. Kim (Eds.) The generative society. Caring for future generations (pp. 35-49). Washington: D.C. American Psychological Association.

Larrain, M., Zegers, B., \& Orellana, Y. (2017). Traducción y adaptación de una escala para evaluar generatividad en abuelos en Santiago de Chile. Psykhé 26(2), 1-18. http://dx.doi.org/10.7764/psykhe.26.2.966

Lehr, U., \& Thomae, H. (2009). Psicología de la senectud: Proceso y aprendizaje del envejecimiento. Barcelona: Herder.

Marín, P. P., Gac, H., \& Carrasco, M. (2006). Escalas de actividades instrumentales de la vida diaria Lawton-Brody. Geriatría y Gerontología (pp. 378-379), (tercera edición ampliada). Santiago: Ediciones Universidad Católica de Chile. 
McAdams, D. P., \& de St. Aubin, E. (1992). A theory of generativity and its assessment through self-report, behavioral acts, and narrative themes in autobiography. Journal of Personality y Social Psychology, 62, 1003-1015. https://doi.org/10.1037/0022-3514.62.6.1003

McAdams, D. P., \& Logan, R. L. (2004). What is generativity? In de E. St. Aubin, D. P. McAdams, \& T. Kim, T. (Eds.), The generative society. Caring for future generations (pp. 15-31). Washington: D.C. American Psychological Association.

McAdams, D. P., de St. Aubin, E., \& Logan, R. L. (1993). Generativity among young, midlife, and older adults. Psychology and Aging, 8, 221-230. http://doi.org/10.1037/0882-7974.8.2.221

Moyano, E., Flores, E., \& Soromaa, H. (2011). Fiabilidad y validez de constructo del test MUNSH para medir felicidad en población de adultos mayores chilenos. Universitas Psychologicas, 10, 567-580.

Muller, Z., \& Litwin, H. (2011). Grandparenting and psychological well-being: how important is grandparent role centrality? European Journal of Ageing, 8, 109-118. http://doi.org/10.1007/s10433-011-0185-5

Muller, Z., \& Litwin, H. (2014). Grandparenting and psychological well-being. In Encyclopedia of Quality of Life and Well-Being Research (pp. 2602-2606). Springer Netherlands.

Neugarten, B. (1974/1996). Age groups in american society and the rise of the young old. In D. A. Neugarten (Ed.). The meanings of age. Selected papers of Bernice L. Neugarten (pp. 34-46). Chicago: The University of Chicago Press.

Neugarten, B., Havighurst, R., \& Tobin, S. (1961). The measurement of life satisfaction. Journal of Gerontology, 16, 134-143. http://dx.doi.org/10.1093/geronj/16.2.134

Neugarten, B., Havighurst, R., \& Tobin, S. (1996). The measurement of life satisfaction. D. A. Neugarten (Ed.) The meanings of age. Selected papers of Bernice L. Neugarten (pp.296-323). Chicago: The University of Chicago Press.

Neugarten, B., \& Weinstein, K. (1964). The changing american grandparent. Journal of Marriage and the Family, 26, 199-204. http://doi.org/10.2307/349727

Peterson, B. (2002). Guarding the next generation: The politics of generative. In E. de St. Aubin, D. P. McAdams, y T. Kim (Eds.). The generative society: Caring for future generations (pp. 195-209). Washington DC, American Psychological Association.

Pinazo, S. (1999). Significado social del rol de abuelo. Revista Multidisciplinar de Gerontología, 9, 169-176. Recuperado de http://nexusediciones.com/pdf/gero1999_3/g-9-3-007.pdf

Pollet, T. V., Nettle, D., \& Nelissen, M. (2007). Maternal grandmothers do go the extra mile: Factoring distance and lineage into differential contact with grandchildren. Evolutionary Psychology, 5, 812-843. http://doi.org/147470490700500412

Powdthavee, N. (2011). Life satisfaction and grandparenthood: Evidence from a nationwide survey. Discussion Paper No. 5869. Bonn: The Institute for the Study of Labor (IZA).

Programa Adulto Mayor UC - Centro UC Estudios Vejez y Envejecimiento (2017). Chile y sus mayores10 años de la Encuesta Calidad de Vida en la Vejez Uc-Caja Los Andes. Santiago, Chile: Caja Los Andes - Pontificia Universidad Católica de Chile.

Reitzes, D. C., \& Mutran, E. J. (2004). Grandparenthood: Factors Influencing Frequency of Grandparent-Grandchildren Contact and Grandparent Role Satisfaction. Journal of Gerontology: Social Sciences, 59B, S9-S16. https://oi. org/10.1093/geronb/59.1.S9

Rodriguez, M. E. (2015). El aprendizaje informal a través de actividades compartidas por abuelos y nietos: La perspectiva de niños chilenos preescolares (Tesis de Magíster en Psicología Educacional). Universidad de los Andes, Santiago, Chile.

Rosenberg, M. (1965). Society and the adolescent self-image. Princeton: Princeton University Press.

Schoklitsch, A., \& Baumann, U. (2011). Measuring generativity in older adults. The development of new scales. The Journal of Gerontopsychology and Geriatric Psychiatry, 24, 31-43. http://doi.org/10.1024/1662-9647/a000030

Silverstein, M., Giarusso, R., \& Bengtson, V. L. (1998). Intergenerational solidarity and the grandparent role. In M. Szinovacz (Ed.). Handbook on grandparenthood (pp. 144-158). Westport, Connecticut, London: Greenwood Press.

Silverstein, M., \& Marenco, A. (2001). How americans enact the grandparent role across the family life course. Journal of Family Issues, 22, 493-522 http://doi.org/10.1177/019251301022004006

Smith, J., Brochelt, M., Maier, H., \& Jopp, D. (2002). Health and well-being in the young old and oldest old. Journal of Social Issues, 58, 715-73. http://doi.org/10.1111/1540-4560.00286

Staudinger, U. M., Fleeson, W., \& Baltes, P. B. (1999). Predictors of subjective physical health and global well-being: Similarities and differences between the United States and Germany. Journal of Personality and Social Psychology, 76, 305-319. http://doi.org/ 10.1037/0022-3514.76.2.305

Stewart, A. J., \& Wandewater, E. A. (1998). The course of generativity. In D. P. McAdams \& E. de St. Aubin (Eds.), Generativity and adult development (pp. 75-100). Washington, DC: APA.

Thiele, D. W., \& Whelan, T. A. (2008). The relationship between grandparent satisfaction, meaning, and generativity. Inter- 
national Journal of Aging and Human Development, 66, 21-48. http:// journals.sagepub.com/doi/abs/10.2190/AG.66.1.b Triadó, C., Martínez, G., \& Villar, F. (2000). El rol y la importancia de los abuelos para sus nietos adolescentes. Anuario de Psicología, 31, 107-118.

Villar, F. (2013). Hacerse bien haciendo el bien: la contribución de la generatividad al estudio del buen envejecer. Informació Psicològica, 104, 39-56.

Zegers, B., Rojas-Barahona, C., \& Förster, C. (2009). Validez y confiabilidad del Índice de Satisfacción Vital (LSI-A) de Neugarten, Havighurst y Tobin en una muestra de adultos y adultos mayores en Chile. Terapia Psicológica, 27, 15-26. http://doi.org/10.4067/S0718-48082009000100002

Tabla 1. Edad promedio (DE) y distribución porcentual según sexo del grupo etario, estado civil, escolaridad, nivel de ingreso, trabajo actual y actividad de voluntariado de la muestra definitiva

\begin{tabular}{|c|c|c|c|c|}
\hline Variable & & $\begin{array}{l}\text { Mujeres } \\
(\mathrm{n}=199)\end{array}$ & $\begin{array}{c}\text { Hombres } \\
(\mathrm{n}=186)\end{array}$ & $\begin{array}{c}\text { Total } \\
(\mathrm{n}=385)\end{array}$ \\
\hline \multirow{2}{*}{ Edad } & Media & 59.69 & 60.70 & 60.18 \\
\hline & (DS) & $(8.37)$ & $(7.54)$ & ( 7.99$)$ \\
\hline \multirow[t]{2}{*}{ Grupo Etario } & $45-59$ & 50.25 & 47.31 & 48.83 \\
\hline & $60-75$ & 49.75 & 52.69 & 51.17 \\
\hline \multirow[t]{4}{*}{ Estado civil } & Casado & 58.29 & 74.19 & 65.97 \\
\hline & Viudo & 15.08 & 2.15 & 8.83 \\
\hline & Soltero & 11.56 & 8.06 & 9.87 \\
\hline & Separado/divorciado & 15.08 & 15.59 & 15.32 \\
\hline \multirow[t]{5}{*}{ Escolaridad } & Ninguna & 0.50 & 0.54 & 0.52 \\
\hline & Básica & 29.65 & 26.88 & 28.31 \\
\hline & Media & 29.65 & 41.40 & 35.32 \\
\hline & Técnica & 17.09 & 6.99 & 12.21 \\
\hline & Universitaria & 23.12 & 24.19 & 23.64 \\
\hline \multirow[t]{3}{*}{ Nivel de Ingreso } & Bajo & 38.24 & 36.79 & 37.53 \\
\hline & Medio & 30.68 & 32.12 & 31.49 \\
\hline & Alto & 30.88 & 31.09 & 30.98 \\
\hline Trabaja actualmente & & 65.33 & 91.40 & 77.92 \\
\hline Realiza voluntariado & & 23.74 & 18.28 & 21.09 \\
\hline
\end{tabular}


Tabla 2.Descripción de la muestra definitiva: Edad promedio (DE) de nietos mayores y menores y distribución porcentual de variables sobre abuelidad según sexo y grupo etario.

\begin{tabular}{|c|c|c|c|c|c|c|c|}
\hline \multirow[b]{2}{*}{ Grupo etario } & \multicolumn{3}{|c|}{ Mujeres } & \multicolumn{3}{|c|}{ Hombres } & \multirow[t]{2}{*}{ Total } \\
\hline & $45-59$ & $60-75$ & Total & 45-59 & $60-75$ & Total & \\
\hline Edad del nieto/a mayor & $\begin{array}{c}7.63 \\
(6.15)\end{array}$ & $\begin{array}{l}17.72 \\
(8.73)\end{array}$ & $\begin{array}{l}12.65 \\
(9.07)\end{array}$ & $7.26(6.06)$ & $\begin{array}{c}13.41 \\
(7.39)\end{array}$ & $10.5(7.44)$ & $\begin{array}{l}11.61 \\
(8.38)\end{array}$ \\
\hline Edad del nieto/a menor & $\begin{array}{c}2.85 \\
(3.14)\end{array}$ & $\begin{array}{c}5.77 \\
(5.17)\end{array}$ & $\begin{array}{c}4.59 \\
(4.68)\end{array}$ & $2.96(2.97)$ & $\begin{array}{c}3.94 \\
(3.89)\end{array}$ & $3.56(3.58)$ & $\begin{array}{c}4.08 \\
(4.20)\end{array}$ \\
\hline $\begin{array}{l}\text { Vive con algún nieto/a } \\
(\%)\end{array}$ & 34.95 & 40.40 & 37.06 & 26.09 & 24.51 & 25.27 & 31.33 \\
\hline \multicolumn{8}{|c|}{ Edad a la que fue abuelo/a (\%) } \\
\hline Menor de 40 años & 26.00 & 15.15 & 20.60 & 19.32 & 7.14 & 11.17 & 16.88 \\
\hline Entre 40 y 59 años & 74.00 & 77.77 & 75.88 & 80.18 & 80.61 & 81.02 & 78.18 \\
\hline Mayor de 59 años & 0.00 & 7.07 & 3.52 & 0.00 & 12.24 & 6.67 & 4.94 \\
\hline
\end{tabular}

Tabla 3 Asociación de las variables edad de nietos mayores y menores y de algunas variables sobre abuelidad con el ingreso familiar de los abuelos/as en la muestra definitiva.

\begin{tabular}{lcccc}
\hline & & Ingreso & & \\
& Alto & Medio & Bajo & P \\
\hline Edad del nieto/a mayor & $13.99^{\mathrm{a}}$ & $13.57^{\mathrm{a}}$ & $8.05^{\mathrm{b}}$ & 0.000 \\
& $(8.36)$ & $(7.93)$ & $(7.53)$ & \\
& & & & 0.000 \\
Edad del nieto/a menor & $2.22^{\mathrm{a}}$ & $5.98^{\mathrm{b}}$ & $4.09^{\mathrm{c}}$ & \\
& $(2.61)$ & $(5.04)$ & $(3.75)$ & \\
& & & & \\
Vive con algún nieto/a (\%) & 18.98 & 48.18 & 30.15 & \\
& & & & \\
Edad a la que fue abuelo/a & & & & \\
Menor de 40 años & 26.05 & 18.33 & 8.22 & \\
Entre 40 y 59 años & 81.43 & 78.34 & 83.56 & \\
Mayor de 59 años & 2.52 & 3.33 & & \\
\hline
\end{tabular}

${ }^{\text {abc }}$ Test de comparación de medias Anova con test post-hoc de Bonferroni. En otro caso Test de Asociación Chi2, $(p<$ $0.05)$ 
Tabla 4. Cuartiles, mínimos y máximos para puntajes EGL-A según sexo, grupo etario y nivel de ingreso en la muestra definitiva

\begin{tabular}{lcccccc}
\hline Variable & $\mathrm{n}$ & Min & Q1 & Q2 & Q3 & Max \\
\hline Sexo & & & & & & \\
Masculino & 186 & 3 & 19 & $23^{* * *}$ & 29 & 39 \\
Femenino & 199 & 7 & 23 & 27 & 31 & 39
\end{tabular}

Grupo etario

40-59

60-75

5

25

39

Nivel de ingreso

1
2
3

$\begin{array}{cccccc}146 & 3 & 19 & 24^{* * *} & 29 & 37 \\ 120 & 10 & 21 & 26 & 31 & 39 \\ 119 & 3 & 22 & 26 & 31 & 39\end{array}$

Trabajan actualmente

No

$\mathrm{Si}$

Viven con algún nieto

No

$\mathrm{Si}$

263

120

3

25

27

137

247

10

3

95

116

174

385

3

3

19

20

22

6

Total

Nota. Test de Kruskal Wallis ${ }^{* * *} \mathrm{p}<0.001,{ }^{* *} \mathrm{p}<0.01,{ }^{*} \mathrm{p}<0.05$

25

27

$20^{* *}$

23

29.5

39

31

39

9

9


Tabla 5. Correlaciones entre las dimensiones de la escala LSI-A y la generatividad positiva según sexo, escolaridad y trabajo actual

\begin{tabular}{|c|c|c|c|c|c|}
\hline & \multicolumn{5}{|c|}{ Dimensiones $L S I-A$} \\
\hline & Congruencia & Entusiasmo & Tono emocional & Autoconcepto & $\begin{array}{r}\text { Resolución y } \\
\text { Fortaleza }\end{array}$ \\
\hline Hombres & 0.23 & 0.03 & -0.06 & -0.06 & 0.05 \\
\hline Básica & 0.05 & 0.04 & 0.07 & -0.29 & -0.03 \\
\hline No Trabaja $(n=43)$ & 0.12 & -0.01 & 0.07 & -0.30 & 0.05 \\
\hline No Trabaja $(n=8)$ & -0.12 & 0.06 & 0.06 & 0.06 & -0.50 \\
\hline Media & 0.35 & 0.15 & 0.09 & 0.07 & 0.09 \\
\hline No trabaja $(n=43)$ & 0.12 & -0.01 & 0.07 & -0.30 & 0.05 \\
\hline Trabaja $(n=76)$ & 0.37 & 0.14 & 0.06 & 0.08 & 0.10 \\
\hline Téc/univ & 0.30 & 0.21 & 0.02 & 0.00 & 0.16 \\
\hline No trabaja $(n=7)$ & -0.48 & -0.03 & 0.00 & -0.14 & - \\
\hline Trabaja $(n=51)$ & 0.36 & 0.23 & 0.03 & 0.03 & 0.18 \\
\hline Mujeres & 0.00 & -0.05 & -0.20 & -0.10 & 0.05 \\
\hline Básica & -0.22 & 0.06 & -0.07 & -0.08 & 0.11 \\
\hline No trabaja $(n=19)$ & -0.05 & 0.18 & 0.11 & 0.27 & 0.38 \\
\hline Trabaja $(n=41)$ & -0.30 & 0.01 & -0.15 & -0.24 & -0.02 \\
\hline Media & 0.17 & 0.04 & -0.06 & -0.1 & 0.12 \\
\hline No trabaja $(\mathrm{n}=22)$ & 0.23 & 0.15 & -0.16 & 0.22 & 0.12 \\
\hline Trabaja $(n=37)$ & 0.13 & -0.01 & 0.01 & -0.25 & 0.10 \\
\hline Téc/univ & 0.16 & -0.11 & -0.22 & -0.15 & -0.08 \\
\hline No trabaja $(n=28)$ & 0.05 & 0.06 & -0.19 & 0.10 & -0.08 \\
\hline Trabaja $(\mathrm{n}=51)$ & 0.21 & -0.16 & -0.21 & -0.31 & -0.06 \\
\hline
\end{tabular}

Nota: Las correlaciones de Spearman más oscuras son significativas $(p<0.05)$ 
